\title{
LA RESPONSABILIDAD SOCIAL EN LAS PYMES DEL SECTOR CALZADO EN ECUADOR
}

María del Carmen Gómez-Romo Universidad Técnica de Ambato Ecuador

Andrés Francisco López-Gómez Universidad Técnica de Ambato Ecuador

Ramiro Patricio Carvajal-Larenas Universidad Técnica de Ambato Ecuador 
Panorama Económico, Vol. 25 - No. 2 (Abril - Junio de 2017), pp. 195-208

María del Carmen Gómez-Romo

Andrés Francisco López-Gómez

Ramiro Patricio Carvajal-Larenas

\title{
La Responsabilidad Social en las Pymes del Sector Calzado en Ecuador
}

\author{
Resumen \\ Esta investigación tiene como propósito determinar el estado de la Responsabilidad Social Empresarial \\ (RSE) en las PyMes del sector manufacturero en la República del Ecuador considerando las siguientes \\ dimensiones: Conocimiento, Ética, Laboral, Ambiental, Filantropía, Comercial, Legal y de Difusión. \\ Se investigó un universo de 85 PyMEs, de donde se extrajo una muestra aleatoria en función de la \\ tasa de respuesta del $28 \%$ con relación al interés en RSE. Se aplicó un cuestionario estructurado y \\ administrado a gerentes o propietarios. Los resultados indican que las dimensiones Comercial, Legal \\ y Ambiental presentan valores promedio ubicándose cerca del nivel "Alto". Las dimensiones Ética, \\ Laboral, Filantrópica y Difusión, se ubican entre el nivel Aceptable y el nivel Alto. Finalmente, la \\ dimensión del Conocimiento se sitúa en el nivel a Aceptable. \\ Palabras clave: Responsabilidad Social Empresarial, PyMEs, Sector Manufacturero. \\ Clasificación JEL: M13, M14, M15, M21
}

\section{Corporate Social Responsability in the Equatorian footwear manufacturing SME sector}

\begin{abstract}
This document aims to analyze Corporate Social Responsability (CSR) in the manufacturing sector of the Republic of Equator, considering the following dimensions: knowledge, ethics, labor, environment, philantropy, sales, legal, and dissemination. For this purpose, 85 manufacturing SMEs were analyzed, taking into account a $28 \%$ of response rate through structured interviews to CEO's. Results indicate that Sales, legal and environmental dimensions are at the highest level. While Ethics, Labor, Philantropy and Dissemination are at acceptable and high levels. Similarly, the Knowledge dimension locates at an acceptable level.

Keywords: Corporate Social Responsability, SMEs, Manufacturing Sector.

JEL Classification: M13, M14, M15, M21
\end{abstract}

\section{Responsabilité sociale des entreprises dans le secteur des PME de la fabrication de chaussures en Equateur}

\section{Résumé}

Ce document vise à analyser la responsabilité sociale des entreprises (RSE) dans le secteur manufacturier de la République de l'Équateur, en prenant en compte les dimensions suivantes: connaissances, éthique, travail, environnement, philantropie, ventes, droit et diffusion. À cette fin, 85 PME manufacturières ont été analysées, en tenant compte d'un taux de réponse de $28 \%$ par le biais d'entretiens structurés avec des chefs d'entreprise. Les résultats indiquent que les dimensions commerciale, juridique et environnementale sont au plus haut niveau. Bien que l'éthique, le travail, la philanthropie et la diffusion soient à des niveaux acceptables et élevés. De même, la dimension Connaissance se situe à un niveau acceptable.

Mots-clés: Responsabilité sociale des entreprises, PME, secteur manufacturier.

Nomenclature JEL: M13, M14, M15, M21 


\section{La Responsabilidad Social en las Pymes del Sector Calzado en Ecuador}

INFORMACIÓN DEL ARTÍCULO

Recepción de artículo: 20/01/2017

Concepto de evaluación: 25/02/2017

Aceptación de artículo: 18/03/2017
María del Carmen Gómez-Romo* Universidad Técnica de Ambato, Ecuador

Andrés Francisco López-Gomez Universidad Técnica de Ambato, Ecuador

Ramiro Patricio Carvajal-Larenas Universidad Técnica de Ambato, Ecuador

\section{INTRODUCCIÓN}

La provincia de Tungurahua está ubicada en la Región Sierra en el Centro del Ecuador, representa aproximadamente el 1,2\% del territorio nacional con una superficie de alrededor $3.400 \mathrm{~km}^{2}$. La población de la provincia, de acuerdo al Censo de Población y vivienda del año 2010, es de 514.354 habitantes, con una densidad poblacional de 149,75 habitantes por $\mathrm{km}^{2}$. El 43\% de la población se encuentra en la zona rural, mientras que el $57 \%$ está en las zonas urbanas. La capital de la provincia es Ambato, que concentra una población de 326.688 habitantes.

La Agenda Tungurahua 2015-2017 describe a la provincia como principalmente agrícola (26,94\% de la población económicamente activa -PEA), manufacturera (18,63\% de la PEA) y comercial al por mayor y menor ( $16,55 \%$ de la PEA), servicios varios (14,22\% de la PEA) y actividades como transporte, construcción, servicios financieros y otros, distribuidas en proporciones menores. Dentro de sus objetivos provinciales se encuentra el incremento del ingreso para cuyo efecto se han definido políticas de fomento productivo que a través de la Estrategia Agropecuaria, la Agenda de Turismo, Agenda de Competitividad y la Agenda Artesanal han direccionado las inversiones del sector público y privado.

Tungurahua, en el año 2014, decide intensificar la matriz productiva, en virtud de sus fortalezas en los ámbitos productivo, agropecuario, comercial e industrial y la demostrada capacidad de sus habitantes para reponerse antes las crisis causadas por catástrofes naturales ocurridas en el pasado, como los terremotos y erupciones volcánicas. La gene-

\footnotetext{
* Autor para correspondencia

Correos electrónicos: mariadgomez@uta.edu.ec*,af.lopez@uta.edu.ec,ramiropcarvajal@uta.edu.ec
} 
ración de ingresos y generación de fuentes de trabajo tiene como base de la pirámide, la transformación y generación de nuevos productos, la sustitución de importación y maximización de exportaciones; entonces reviste gran importancia, transitar de ser productores de materias primas a productores de productos terminados creando valor agregado y optimizando recursos, bajo parámetros socialmente responsables.

La acción del Gobierno Provincial de Tungurahua ha girado sobre grandes objetivos trazados en la Agenda de Tungurahua, desde el año 2003 "agua, gente y trabajo", que bajo principios de "representatividad, corresponsabilidad y responsabilidad", ha consolidado un Modelo de Gestión que ha permitido promover el desarrollo de la provincia. El Ministerio de Coordinación de la Producción, Empleo y Competitividad, en la Agenda para la Transformación Productiva Territorial de Tungurahua (2011, pag. 9), manifiesta que las "mipymes tienen una importancia estratégica en el crecimiento de la economía para el desarrollo del aparato productivo local, y la mejor posición competitiva del país". Como se sabe, al decir "mipymes", se involucra a las micro, pequeñas y medianas empresas, de entre las cuales la mayor parte corresponden a microempresas (menos de 10 empleados) y son las pequeñas y las medianas las que presentan una mejor organización para desarrollar actividades de Responsabilidad Social.

La provincia de Tungurahua tiene una presencia muy fuerte en cuanto a la producción de calzado, entonces, resulta interesante mencionar que a pesar de que esta provincia representa, aproximadamente, el 1,2\% de la extensión del territorio nacional $\left(3.400 \mathrm{~km}^{2}\right.$ de $\left.283.560 \mathrm{~km}^{2}\right)$, produce alrededor del 50\% de la producción nacional. El gobierno provincial de Tungura- hua, con el propósito de dar cumplimiento al objetivo estratégico:

Estimular la actividad económica local
mediante procesos participativos de
actores públicos y privados, el desa-
rrollo de ventajas comparativas locales
para propiciar un clima de negocios
que favorezca, la creación de valor
agregado, fomento de las exportacio-
nes, el mejoramiento de la productivi-
dad, la competitividad y la creación de
empleo y de ingresos. (Agenda Tungu-
rahua 2015-2017, pg 19)

Propone elaborar un proyecto, bajo el slogan, "Un pueblo un Producto", para asociar a los productores de calzado, para cuya implementación es menester que se delinean prácticas de Responsabilidad Social Empresarial.

El Sector Calzado de la provincia, en estos últimos años, de acuerdo al Ministerio de Industrias y Productividad, ha tenido un importante crecimiento, así en el año 2008, la venta interna de calzado nacional fue de 165 millones de dólares, mientras que en el año 2011, ascendió a 318 millones de dólares. En el mismo sentido, la Cámara Nacional de Calzado, señala que en el año 2008 , la producción de calzado fue de 15 millones de pares de zapatos, según lo publicado en la Revisa Líderes, en el diario El Comercio, (2012). Como se puede apreciar, el crecimiento del sector calzado en la provincia de Tungurahua, en tres años, fue evidente, incrementando tanto su producción como sus ventas alrededor del $92 \%$, lo que se explica por las políticas arancelarias del gobierno ecuatoriano implementadas en esa época, consistentes en un gravamen de 6.00 USD más el $10 \%$ ad valorem a cada par de zapatos importado, lo que dinamizó la producción y ventas de calzado al interior del país. 
La presidenta de la Cámara Nacional de Calzado (CALTU), Lilia Villavicencio, en declaraciones de prensa publicadas en el Diario Nacional La Hora, el 03 de Abril del presente año, manifestó que el Sector Calzado, hasta el 2013, había crecido en un 80\%; mientras que del 2014 al 2015 tuvo un decrecimiento del $30 \%$, por cuyo efecto, cerraron 187 unidades productivas en el 2015. Del mismo modo, el 09 de Abril, en la misma fuente se da a conocer que la disminución de ventas y el incremento de la cartera vencida, al primer trimestre del 2016, ha dado lugar al cierre de nuevas unidades de producción de calzado. Como se puede apreciar, la situación descrita es una situación alarmante, sin embargo, puede convertirse en una oportunidad de hacer las cosas de una manera diferente y mejorada con la inclusión de acciones de Responsabilidad Social Empresarial con impacto en los diversos stackeholders.

En este sentido, la presente investigación, proporcionará información de línea base para describir el estado en que se encuentran las prácticas de Responsabilidad Social Empresarial (RSE) en las PyMes del Sector Calzado a fin de contribuir con elementos de juicio que propicien su desarrollo dentro de la gestión administrativa, en razón de que Responsabilidad Social Empresarial constituye una valiosa estrategia que a través de mecanismos factibles de ser aplicados contribuya al Sector dentro del contexto donde se desarrolla.

\section{LAS PEQUEÑAS Y MEDIANAS EMPRESAS (PYMES)}

La presencia de las PyMes en América Latina tiene un alto impacto en la economía de la región, siendo una fuente importante de ingresos y empleo. De acuerdo a promedios reportados por el Banco Mundial, las PyMes en América
Latina generan el alrededor del 60\% de empleo y el $30 \%$ del producto interno bruto, (PIB), mientras que los países de la Organización para la Cooperación y el Desarrollo Económicos (OCDE) generan alrededor del $60 \%$ del PIB y entre 50 y 60\% de empleo, observándose que a pesar de que la proporción de generación de empleo es similar, las PyMes del segundo grupo, prácticamente duplican el aporte del PIB, de las primeras. De lo manifestado se concluye que, que en América Latina la contribución de las PyMes es notable, sin embargo de no estar muy bien organizadas ni potenciadas, es difícil su sostenibilidad. Al hacer una revisión de éstas, se puede apreciar una evidente heterogeneidad en los diferentes sectores productivos, en cuanto a su estructura, su nivel de productividad y competitividad, lo que se explica por las insuficientes políticas y programas de impulso a la creación y desarrollo de la pequeña y mediana empresa. Por otro lado, solamente el 30\% del producto interno bruto regional, PIB, proviene de las PyMes, frente al 70\% proveniente de grandes empresas, CEPAL, (2013), con lo que se puede apreciar que aunque se trate de un gran número de PyMes, su aporte al PIB no guarda la misma proporción, pues las PyMes siendo mucho más numerosas, aportan con una proporción menor.

En América Latina las PyMes enfrentan problemas relativos a la situación sociopolítica que les ha tocado vivir a través de los distintos gobiernos, como lo relata Pérez, R (2015); así, los años 70 se caracterizados por dictaduras, seguidas por la social democracia vivida en la década de los 80 , mientras que en los años 90 hubo una marcada tendencia derechista; al iniciar el siglo XXI, emergió una nueva izquierda que ha generado bastante insatisfacción; finalmente, 
en la segunda década aparecen serias posibilidades de cambio de dirección de la situación sociopolítica en América Latina.

Es en el escenario descrito anteriormente que las PyMes han tenido un apoyo limitado para su desarrollo, más aún, a pesar de que en décadas anteriores hubieron determinadas políticas que produjeron viento a su favor, hoy en día, están atravesando dificultades, dada su pérdida de competitividad, pues, como es conocido, la productividad de las Pymes está entre el $25 \%$ y el $50 \%$ de las empresas grandes, razón por la cual se deben buscar alternativas para su resurgimiento y desarrollo.

En Ecuador, un estudio realizado por la FLACSO (2013), expresa la importancia de las PyMes en el tejido empresarial ecuatoriano, tal que, el Ministro de Industrias y Productividad, extiende una invitación a inversionistas, académicos, emprendedores y sector productivo en general, a tomar acciones para mejorar la situación de las MiPyMes en el desarrollo del país, en tal virtud la implementación de buenas prácticas en hacer negocios facilita a la consecución de una ventaja competitiva.

\section{LA RESPONSABILIDAD SOCIAL.}

\subsection{La conciencia empresarial en la Responsabilidad Social.}

Las empresas en la actualidad han empezado a concientizar sus acciones enfocándose no solo en la generación de recursos económicos, sino también en la generación de bienestar social y ambiental. Para López (2013, p. 39) la Responsabilidad Social Empresarial es una filosofía que ha obligado a las empresas a integrar en su visión el compromiso hacia la sociedad, a través de una nueva forma de hacer negocios donde no sólo importa cuánto gano, sino también cómo lo gano. Por lo tanto, para que las empresas sean económicamente sustentables y logren utilidades duraderas deben considerar el aspecto social, ambiental y económico en sus acciones. (Aquino, Reficco, \& Arroyo, 2014; Crespo, 2010)

En América Latina, la RSE se encuentra aún en una fase de desarrollo, análisis y exploración (López, 2013). En el contexto ecuatoriano la investigación llevada a cabo por Torresano (2012), afirma que cerca de la mitad de las empresas ha introducido temas relacionados con RSE (51\%), el $80 \%$ de ellas en la última década. Del $49 \%$ de empresas que aún no implementan una estrategia o aspectos de la responsabilidad social, al 56\% le interesa la temática.

Ante esta realidad, se propone identificar el nivel de responsabilidad social implementado en las PyMes del Sector Calzado de la provincia de Tungurahua tomando en cuenta que no se ha encontrado difusión de sus actividades socialmente responsables en el caso de que hayan sido llevada a cabo, de allí que la existencia de memorias sostenibles es una necesidad para divulgar información requerida por la sociedad en cuanto a la actuación no-económica por parte de la organización (Bauber \& Fenin, 1973), de hecho los diferentes grupos de interés deben ser informados sobre las actividades que las empresas llevan a cabo sea en cuanto a lo social como a lo ambiental.

\subsection{Beneficios de la Responsabilidad Social Empresarial.}

La responsabilidad social no solo se refiere a ser social y ambientalmente responsable. Sino que, internamente la empresa no puede ser indiferente a los problemas 
de la sociedad (Vives, 2013). Desde un punto de vista positivo la responsabilidad social tiene impacto en varios aspectos que son fuentes de ventaja competitiva en las empresas: estructura de costes, recursos humanos, clientes, innovación, gestión de riesgos, reputación y resultados financieros (Corrales, 2011). Siendo así, la responsabilidad social engloba las decisiones, las medidas, las actividades y las acciones éticas y transparentes realizadas por cualquier empresa durante el desarrollo de su actividad económica. A la vez, se puede lograr un aumento de la eficacia, mejora de la imagen de la empresa, atracción y retención del talento, aumento de la lealtad de los clientes, etc., y en definitiva, un impacto positivo en sus resultados (Benito \& Esteban, 2010). En definitiva, se convierte en una empresa más rentable en el largo plazo (Ekos Negocios, 2011). Obtener los beneficios antes indicados es de mucho interés en el Sector Calzado para cuyo propósito es menester alinear tanto las políticas como las estrategias tendientes al cumplimiento de actividades de Responsabilidad Social en sus distintas dimensiones.

Existe un amplio sustento bibliográfico en torno al concepto y a las dimensiones de la Responsabilidad Social Empresarial (RSE) dentro de cuyo panorama se encuentran las dimensiones económicas, legales, éticas y filantrópicas que se interrelacionan unas con otras evidenciando que la RSE tiene una carácter multidimensional, Alvarado (2008). En la dimensión económica se espera que las empresas sean rentables y generen utilidades en función de la producción de bienes o servicios. La dimensión legal, prevé que todas las actividades económicas se ejecuten en sujeción al marco jurídico. La dimensión ética exige el cumplimento de aspectos éticos mediante prácticas empresariales que satisfagan a la sociedad al amparo de principios y normas éticas. Finalmente la dimensión filantrópica despliega una amplia gama de oportunidades que pueden dar lugar a distintos tipos de acciones que queda a criterio de las empresas adoptarlas; en este sentido, las empresas pueden tomar la decisión de involucrarse en actividades de interés social, por cuya participación la sociedad les brinde reconocimiento.

Si bien las PyMes en América Latina, por un lado, tienen una presencia notable, por otro lado, tienen una gran necesidad de desarrollarse y competir, así la participación en actividades de Responsabilidad, aparecer como una importante oportunidad para posicionarse frente a la comunidad, como lo resume Aguilera \& Puerto (2012):

La responsabilidad social empresarial actúa como un agente clave que impulsa el crecimiento de la empresa. El crecimiento empresarial es una variable que depende de numerosos factores; la responsabilidad social empresarial es uno de ellos, ya que favorece toda iniciativa que se origina en la empresa, facilitando el posicionamiento de su marca, renovando la imagen corporativa, capturando la preferencia y la lealtad de los clientes y promoviendo la perfecta armonía entre la empresa y la comunidad en la que opera. En este contexto, la responsabilidad social empresarial activa el crecimiento de la empresa, debido a que mejora ostensiblemente la reputación y la credibilidad, y con ello logra el reconocimiento ante sus grupos de interés (stakeholder), especialmente cuando el compromiso con el desarrollo de la estrategia de responsabilidad social es percibido como reflexivo, ético y espontáneo. 
Investigar acerca de la Responsabilidad Social Empresarial en las PyMes de los diversos sectores productivos en Ecuador, dota de información relevante para establecer una línea base a partir de la cual se puede potencia su desarrollo. "La RSE ha comenzado a visualizarse como un elemento clave de la gestión empresarial que afecta directamente en los resultados del negocioy por ende, la competitividad de las empresas, sean éstas grandes, medianas o pequeñas." Belmar. (2009, p. 7). De modo que, su aplicación es independiente del tamaño de las empresas y su apliación está en función de los criterios, y decisiones de los empresarios, que con un compromiso sostenido, permitan otenener beneficios visibles tanto para la empresa como para la sociedad.

\section{METODOLOGÍA}

Esta investigación tiene un enfoque cualitativo y cuantitativo con una modalidad de campo y documental aplicada a nivel descriptivo. De acuerdo a la información obtenida del Instituto Nacional de Estadísticas y Censos (INEC, 2014), extraída del REDATAM (Recuperación de Datos para Áreas Pequeñas),elsector Calzadoenlaprovincia de Tungurahua está compuesto por 642 microempresas, 71 empresas pequeñas, 14 empresas medianas y 4 empresas grandes. Por lo indicado, el universo del presente estudio está constituido por 85 PyMEs de calzado, de donde se extrajo una muestra aleatoria de 24 PyMes en función de la tasa de respuesta del $28 \%$ con relación al interés en RSE. Se aplicó un cuestionario administrado a gerentes o propietarios. Para la aplicación el cuestionario estructurado de 23 items se utilizó la escala de Likert de 1 al 5, con la siguiente equivalencia: 1 Ninguno. 2 Bajo. 3 Aceptable. 4 Alto. 5 Muy alto, a través de una encuesta administrada a los gerentes o propietarios de las Pymes del Sector Calzado para determinar el estado de Responsabilidad Social en cuanto a los siguientes dimensiones: Conocimiento, Ética, Laboral, Ambiental, Filantropía, Comercial, Legal y de Difusión. Los datos obtenidos fueron tratados con el Statistical Package for the Social Sciences, SPSS, (Arbuckle, 2010), de donde se extrajeron los valores máximos, mínimos, promedio y desviación típica (Valenzuela, 2014) de los valores obtenidos en cada pregunta al interior de cada una de las dimensiones, así como el coeficiente de variación que es la razón entre la desviación típica y el promedio.

\section{RESULTADOS.}

Los resultados se reportan de manera detallada en las siguientes tablas y gráfico de radar con el promedio resultante en cada dimensión. 
Tabla N¹. Análisis Descriptivo de las prácticas de Responsabilidad Social dentro de las dimensiones Conocimiento, Ética, Laboral, Ambiental, Filantropía, Comercial, Legal y de Difusión.

\begin{tabular}{|c|c|c|c|c|c|c|}
\hline $\mathbf{N}^{\circ}$ & VARIABLE & TOTAL & MÍNIMO & MÁXIO & MEDIA & $\begin{array}{l}\text { DESVIACIÓN } \\
\text { TÍPICA }\end{array}$ \\
\hline 1 & $\begin{array}{l}\text { ¿Cuál es el grado de } \\
\text { conocimiento de sus } \\
\text { colaboradores en temas } \\
\text { de Responsabilidad Social } \\
\text { Empresarial? } \\
\end{array}$ & 24 & 1 & 5 & 3,13 & 1,116 \\
\hline 2 & $\begin{array}{l}\text { ¿En la empresa se han } \\
\text { definido claramente cuáles } \\
\text { son los valores que guían su } \\
\text { accionar? }\end{array}$ & 24 & 2 & 5 & 3,54 & 1,021 \\
\hline 3 & $\begin{array}{l}\text { ¿Cómo calificaría las } \\
\text { prácticas de competencia y } \\
\text { negociación justa entre su } \\
\text { empresa y las de su sector? }\end{array}$ & 24 & 1 & 5 & 3,58 & 1,176 \\
\hline 4 & $\begin{array}{l}\text { Cómo calificaría el precio de } \\
\text { sus productos/servicios con } \\
\text { relación a la calidad ofrecida } \\
\text { a sus clientes? }\end{array}$ & 24 & 0 & 5 & 3,67 & 1,204 \\
\hline 5 & $\begin{array}{l}\text { ¿Cómo calificaría la } \\
\text { participación de sus clientes, } \\
\text { socios, proveedores y } \\
\text { empleados con los valores de } \\
\text { su empresa? }\end{array}$ & 24 & 1 & 5 & 3,54 & ,833 \\
\hline 6 & \begin{tabular}{|l|} 
¿Cómo calificaría la \\
contribución de su empresa \\
al desarrollo y capacitación \\
continua de sus trabajadores?
\end{tabular} & 24 & 2 & 5 & 3,88 & ,900 \\
\hline 7 & $\begin{array}{l}\text { ¿Cómo evaluaría } \\
\text { la participación de } \\
\text { los empleados en la } \\
\text { Responsabilidad Social de su } \\
\text { empresa? }\end{array}$ & 24 & 1 & 5 & 3,58 & ,881 \\
\hline 8 & $\begin{array}{l}\text { ¿En qué medida considera } \\
\text { que la empresa incentiva a } \\
\text { sus empleados por el correcto } \\
\text { desempeño laboral? }\end{array}$ & 24 & 1 & 5 & 3,25 & 1,073 \\
\hline 9 & $\begin{array}{l}\text { ¿En qué grado la empresa } \\
\text { ha desarrollado prácticas } \\
\text { relacionadas con el manejo } \\
\text { de residuos o desechos? }\end{array}$ & 24 & 1 & 5 & 3,42 & 1,139 \\
\hline 10 & $\begin{array}{l}\text { ¿En qué medida la empresa } \\
\text { ha invertido en equipos, } \\
\text { maquinarias y procesos } \\
\text { que disminuyan el impacto } \\
\text { ambiental? }\end{array}$ & 24 & 2 & 5 & 3,83 & ,816 \\
\hline 11 & $\begin{array}{l}\text { ¿En qué nivel su empresa } \\
\text { cumple con el cuidado del } \\
\text { medio ambiente? }\end{array}$ & 24 & 2 & 5 & 3,92 & ,830 \\
\hline 12 & $\begin{array}{l}\text { ¿Cómo evaluaría las } \\
\text { actividades de su empresa al } \\
\text { beneficio de la comunidad? }\end{array}$ & 24 & 2 & 5 & 3,75 & ,794 \\
\hline
\end{tabular}




\begin{tabular}{|c|c|c|c|c|c|c|}
\hline 13 & $\begin{array}{l}\text { ¿Cómo calificaría el diálogo } \\
\text { de su empresa sobre temas } \\
\text { delicados, polémicos o } \\
\text { negativos que afecten a la } \\
\text { comunidad? }\end{array}$ & 24 & 2 & 5 & 3,54 & 833, \\
\hline 14 & \begin{tabular}{|l|} 
¿Cómo calificaría las \\
actividades realizadas por \\
su empresa destinadas a \\
patrocinios, donaciones, \\
financiación de proyectos, \\
voluntariado, entre otros? \\
\end{tabular} & 24 & 2 & 5 & 3,46 & 1,062 \\
\hline 15 & $\begin{array}{l}\text { ¿Cómo calificaría el } \\
\text { reconocimiento que recibe su } \\
\text { empresa de la comunidad por } \\
\text { las actividades sociales que } \\
\text { realiza? }\end{array}$ & 24 & 1 & 5 & 3,29 & 1,122 \\
\hline 16 & $\begin{array}{l}\text { ¿En qué medida, proporciona } \\
\text { su empresa información de } \\
\text { los riesgos y beneficios de } \\
\text { sus productos/servicios que } \\
\text { ofrece a sus clientes y su } \\
\text { comunidad? }\end{array}$ & 24 & 2 & 5 & 3,58 & 830, \\
\hline 17 & $\begin{array}{l}\text { ¿Cómo evaluaría el } \\
\text { cumplimiento de su } \\
\text { empresa para satisfacer las } \\
\text { necesidades de sus clientes? }\end{array}$ & 24 & 3 & 5 & 3,83 & ,761 \\
\hline 18 & $\begin{array}{l}\text { ¿En qué grado la empresa } \\
\text { recoge las sugerencias y } \\
\text { reclamos de los clientes o de } \\
\text { la comunidad en general? }\end{array}$ & 24 & 2 & 5 & 3,83 & 868 \\
\hline 19 & $\begin{array}{l}\text { Con respecto a la cultura } \\
\text { empresarial social, ¿en qué } \\
\text { medida considera que los } \\
\text { trabajadores han aportado a } \\
\text { su empresa? }\end{array}$ & 24 & 1 & 5 & 3,38 & 1,135 \\
\hline 20 & $\begin{array}{l}\text { ¿En qué grado los directivos } \\
\text { y trabajadores de la } \\
\text { empresa dan importancia } \\
\text { a las actividades de } \\
\text { Responsabilidad Social? } \\
\end{array}$ & 24 & 2 & 5 & 3,58 & ,776 \\
\hline 21 & $\begin{array}{l}\text { ¿En qué medida considera } \\
\text { que su empresa cumple } \\
\text { con las regulaciones } \\
\text { gubernamentales? }\end{array}$ & 24 & 3 & 5 & 4,29 & 624 \\
\hline 22 & $\begin{array}{l}\text { ¿Cómo calificaría el grado de } \\
\text { información difundido a la } \\
\text { comunidad o rendición de } \\
\text { cuentas a la sociedad? }\end{array}$ & 24 & 0 & 5 & 3,52 & 1,085 \\
\hline 23 & $\begin{array}{l}\text { ¿En qué medida informa } \\
\text { su organización sobre } \\
\text { la responsabilidad } \\
\text { social empresarial a sus } \\
\text { socios estratégicos y a la } \\
\text { comunidad? }\end{array}$ & 24 & 0 & 5 & 3,48 & 1,159 \\
\hline
\end{tabular}

Fuente: Encuesta 
Se observa que el "cumplimiento de regulaciones gubernamentales" (ítem 21) presenta el promedio más alto de todos que corresponde a 4,29, lo que significa que está sobre el nivel Alto, su coeficiente de variación es de $14,5 \%$, reflejando que las respuestas tuvieron una escasa variación con respecto a la media de esta dimensión. En cuanto al cuidado del medio ambiente, el promedio es de 3,92, que india que está muy cerca del nivel Alto, y un coeficiente de variación de 21,2 \% que indica que la variación de las respuestas con respecto a la media es inferior al primer cuartil.

Al contrario, el grado de conocimiento de los colaboradores en temas de Responsabilidad Social Empresarial, reporta el promedio más bajo, con 3,13, que refleja un nivel Aceptable con coeficiente de variación de $35,7 \%$ que revela una significativa variabilidad de las respuestas de con respecto al promedio. El valor anterior es seguido por el de reconocimiento que recibe la empresa por parte de la comunidad por las acti- vidades sociales de responsabilidad social que realiza la empresa, que tiene un promedio de 3,29 que describe un nivel Aceptable, con un coeficiente de variación de $34,1 \%$; caso que es ligeramente superior al anterior. (Tabla No.2)

Las dimensiones Comercial, Legal y Ambiental reportan el promedio más alto, que está alrededor de 3,75, cuya equivalencia en la escala de Likert se acerca al nivel "Alto".

Las dimensiones Ética, Laboral, Filantrópica y Difusión, con promedios 3,58; 3,57; 3,51 y 3,50 respectivamente, reflejan un nivel intermedio entre el nivel Aceptable y Alto.

Finalmente la dimensión del Conocimiento se ubica en el nivel más bajo que corresponde a Aceptable.

Lo indicado anteriormente se aprecia en el gráfico de radar que a continuación se presenta (Figura No 1).

Tabla N². Resumen del Análisis Descriptivo de las prácticas de Responsabilidad Social dentro de las dimensiones Conocimiento, Ética, Laboral, Ambiental, Filantropía, Comercial, Legal y de Difusión.

\begin{tabular}{|c|c|c|c|c|}
\hline DIMENSIÓN & MÍNIMO & MÁXIO & MEDIA & $\begin{array}{c}\text { DESVIACIÓN } \\
\text { TÍPICA }\end{array}$ \\
\hline Conocimiento & 1,00 & 5,00 & 3,13 & 1,12 \\
\hline Ética & 1,00 & 5,00 & 3,58 & 1,06 \\
\hline Laboral & 1,33 & 5,00 & 3,57 & 0,95 \\
\hline Ambiental & 1,67 & 5,00 & 3,72 & 0,93 \\
\hline Filantrópica & 1,75 & 5,00 & 3,51 & 0,95 \\
\hline Comercial & 2,33 & 5,00 & 3,75 & 0,82 \\
\hline Legal & 2,00 & 5,00 & 3,75 & 0,85 \\
\hline Difusión & 0,00 & 5,00 & 3,50 & 1,12 \\
\hline
\end{tabular}

Fuente: Tabla 1 


\section{Figura No 1.}

\section{Responsabilidad Social en las PyMes de Calzado de Tungurahua}

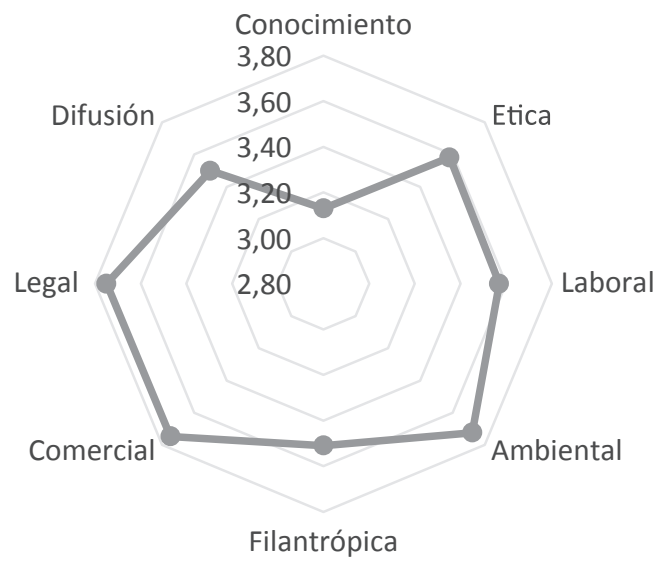

Fuente: Tabla 2.

\section{CONCLUSIONES}

En estos últimos años se ha observado una mayor participación en prácticas de RSE por parte de las empresas grandes. Esto ha tenido una importante influencia en las empresas medianas y pequeñas, cuyo impacto podrá ser demostrado de manera contundente en el tiempo.

De acuerdo a los resultados de esta investigación, en opinión de los operarios o gerentes de la PyMes de calzado, las prácticas de RSE se la lleva a cabo a un nivel entre aceptable y alto; sin embargo, leyendo más allá de los números, a partir de los resultados de esta investigación, no se podría afirmar de manera definitiva que se trata de una verdadera realización de prácticas de RSE en cada una de las dimensiones estudiadas, para lo cual, sin duda se hace necesario complementar la investigación desde otras perspectivas tales como la de los trabajadores, de la comunidad y de las evidencias palpabl e Por otra parte, conociendo el contexto de las PyMes del sector en la provincia, se puede afirmar, que existe un importante interés y motivación en los empresarios para incursionar en estas prácticas, revelando además que le han otorgado un alto grado de importancia a este tema.

Siendo el valor más bajo el obtenido en la dimensión del Conocimiento, se concluye que esta dimensión abre la oportunidad de trabajar ampliamente en la gestión del conocimiento aplicado a las prácticas de la RSE, pudiéndose constituir esta dimensión en la clave para potenciar a todas las demás dimensiones de la RSE, debiendo gestionar el conocimiento de modo sostenido y con la participación de todos los stakeholders. 


\section{REFERENCIAS BIBLIOGRÁFICAS}

Arbuckle, J. (2010). IBM SPSS Amos 19 user's guide. Crawfordville, FL: Amos Development Corporation, 635.

Alvarado, A., \& Sclesinger, M. (Septiembre de 2008). Dimensionalidad de la Responsabilidad Social empresarial percibida y sus efectos sobre la imagen y la Reputación: una aproximación desde el modelo de Carroll. Estudios Gerenciales, 24(108), 37-59.

Aguilera , A., \& Puerto , D. (2012). Crecimiento empresarial basado en la Responsabilidad Social. Pensamiento \& Gestiòn, 1. Obtenido de http://www.scielo.org.co/scielo.php?script=sci_ arttext\&pid=S165762762012000100002\&lang=pt

Aquino Alves, M., Reficco, E., \& Arroyo, J. (2014). Perspectivas sobre la situación y proyección de la responsabilidad social empresarial en América Latina. Revista de Administración de Empresas, 54(1), 10-11.

Benito, S., \& Esteban, P. (2010). La influencia de las políticas de responsabilidad social y la pertenencia a redes de cooperación en el capital relacional y estructural de las microempresas. Universidad Europea de Madrid, 1-29.

Belmar, C. (2009). Situación de la Responsabilidad Social Empresarial de la Pyme en Bolivia. FUNDES, 1-59.

Corrales, L. (2011). La Responsabilidad Social Empresarial en la pequeña y mediana empresa Guía de buenas prácticas. Proyecto IMPULSA RSE-PYME, 1-119. Cuadernos de Gestión , 3.

Ekos Negocios. (2011). Empresas con Responsabilidad Corporativa. Ekos RSE, 1-146.

H. Gobierno Provincial de Tungurahua. 2015. Agenda Tungurahua 2015-2017. Ambato: Mundo Gráfico.

INEC. 2014. redatam.inec.gob.ec. Recuperación Datos para Áreas pequeñas por Microcomputador. REDATAM Último acceso: 18 de Septiembre de 2016. http://redatam.inec.gob.ec/cgibin/RpWebEngine.exe/EasyCross?\&BASE=DIEE2014\&ITEM=CRUZSIMPLE\&MAIN=WebServerMain.inl.

La Hora. (2016). Artesanos de Calzado Cierran sus Talleres. Recuperado de: https://issuu.com/LaHora/ docs/ambato09abril2016. Pg. A3.

La Hora. (2016). 187 empresas de calzado cerraron sus negocios. Recuperado de: https://issuu.com/ LaHora/docs/ambato03abril2016. Pg. B6.

López, A. (2013). Hacia la responsabilidad social empresarial de pequeñas empresas: caso México. Revista Internacional Administración \& Finanzas, 6(6), 39-54.

Ministerio de Coordinación de la Producción, Empleo y Competitividad. (Junio de 2011). Plan de Mejora Competitiva Cuero y Calzado. Obtenido de http://es.slideshare.net/mcpec1/plan-demejora-competitiva-cuero-y-calzado

Pérez, R. (2015). América Latina. El retorno de la derecha o el retorno de la antipolítica. Listin Diario. Santo Domingo. República Dominicana.

Revista Líderes. (22 de Octubre de 2012). La producción de calzado pisa fuerte en el país. Revista Líderes. Obtenido de http://www.revistalideres.ec/lideres/produccion-calzado-pisa-fuerte-pais. html 
Torresano, M. (2012). Estudio de Responsabilidad Social de Empresas del Ecuador. EKOS, 5-63.

Valenzuela, L. J. (2014). Prácticas de Responsabilidad Social, Reputación Corporativa y Desempeño Financiero. Revista de Administración de Empresas., 329-343.

Vives, A. (2013). Empleo y emprendimiento como responsabilidad social de las empresas. Revisa Journal Georgetown University, 1-19.

\section{Para citaciones:}

Gómez-Romo, M., López-Gómez, A. F., \& Carvajal-Larenas, R. P. (2017). La Responsabilidad Social en las Pymes del Sector Calzado en Ecuador. Panorama Económico, 25, 2, pp. 195-208.

\section{AUTORES}

María del Carmen Gómez-Romo

Docente e Investigadora de la Universidad Técnica de Ambato (Ecuador). Candidata a Doctor en Administración de la Universidad Nacional de Rosario (Republica Argentina) y Magister en Gerencia Empresarial en la Escuela Politecnica Nacional (Ecuador)

\section{Andrés Francisco López-Gomez}

Docente e Investigador de la Universidad Técnica de Ambato (Ecuador). Magister en Administración de Empresas mención Planeación de la Pontifica Universidad Católica del Ecuador.

\section{Ramiro Patricio Carvajal-Larenas}

Docente e Investigador de la Universidad Técnica de Ambato (Ecuador). Doctor en Economía y Dirección de Empresas de la Universidad de Deusto-Campus de San Sebastián en el País Vasco (España). Actualmente Decano de la Facultad de Ciencias Administrativas de la Universidad Técnica de Ambato. 